\title{
A PROGRADE, LOW-INCLINATION ORBIT FOR THE VERY HOT JUPITER WASP-3b*
}

\author{
Anjali Tripathi ${ }^{1,6}$, Joshua N. Winn ${ }^{1}$, John Asher Johnson ${ }^{2,7}$, Andrew W. Howard ${ }^{3}$, Sam Halverson ${ }^{3}$, \\ Geoffrey W. Marcy ${ }^{3}$, Matthew J. Holman ${ }^{4}$, Katherine R. De Kleer ${ }^{1}$, Joshua A. Carter ${ }^{1}$, Gilbert A. Esquerdo ${ }^{4}$, \\ Mark E. EveretT ${ }^{5}$, AND Nicole E. CABrera ${ }^{2,8}$ \\ ${ }^{1}$ Department of Physics, and Kavli Institute for Astrophysics and Space Research, Massachusetts Institute of Technology, Cambridge, MA 02139, USA; \\ tripathi@mit.edu, jwinn@mit.edu \\ 2 Institute for Astronomy, University of Hawaii, Honolulu, HI 96822, USA \\ ${ }^{3}$ Department of Astronomy, University of California, Mail Code 3411, Berkeley, CA 94720, USA \\ ${ }^{4}$ Harvard-Smithsonian Center for Astrophysics, 60 Garden Street, Cambridge, MA 02138, USA \\ ${ }^{5}$ Planetary Science Institute, 1700 East Fort Lowell Road, Suite 106, Tucson, AZ 85719, USA \\ Received 2009 November 20; accepted 2010 April 3; published 2010 April 28
}

\begin{abstract}
We present new spectroscopic and photometric observations of the transiting exoplanetary system WASP-3. Spectra obtained during two separate transits exhibit the Rossiter-McLaughlin (RM) effect and allow us to estimate the sky-projected angle between the planetary orbital axis and the stellar rotation axis, $\lambda=3.3_{-4.4}^{+2.5} \mathrm{deg}$. This alignment between the axes suggests that WASP-3b has a low orbital inclination relative to the equatorial plane of its parent star. During our first night of spectroscopic measurements, we observed an unexpected redshift briefly exceeding the expected sum of the orbital and RM velocities by $140 \mathrm{~m} \mathrm{~s}^{-1}$. This anomaly could represent the occultation of material erupting from the stellar photosphere, although it is more likely to be an artifact caused by moonlight scattered into the spectrograph.
\end{abstract}

Key words: planetary systems - stars: activity - stars: individual (WASP-3) - techniques: radial velocities

Online-only material: machine-readable table

\section{INTRODUCTION}

One of the hottest exoplanets yet discovered is WASP-3b, a giant planet with a mass of $1.76 M_{\mathrm{J}}$ that orbits an F7-8V star at a distance of only $0.032 \mathrm{AU}$, giving the planet an equilibrium temperature of $1960 \mathrm{~K}$ (Pollacco et al. 2008). This close-in, transiting system is an ideal target for study with the Rossiter-McLaughlin (RM) effect because the host star is both relatively bright $(V=10.5)$ and rapidly rotating ( $v \sin i_{\star}=13.4 \pm 1.5 \mathrm{~km} \mathrm{~s}^{-1}$ ), and the transit occurs with an impact parameter near 0.5 . Observations of the RM effect yield valuable information about the system, namely, the angle $(\lambda)$ between the sky projections of the planetary orbital axis and the stellar rotation axis. This parameter is a basic geometric property of the system and a possible clue about the processes of planet formation and orbital migration. Detailed descriptions of the RM effect and its applications can be found in Ohta et al. (2005), Gimenez (2006), Gaudi \& Winn (2007), and Fabrycky \& Winn (2009), while early examples of such measurements for exoplanetary systems are given by Queloz et al. (2000) and Winn et al. (2005).

In this paper, we report the results of new photometric and spectroscopic observations of WASP-3, which were conducted with the primary goal of determining $\lambda$ and the secondary goal of refining estimates of other system parameters. In Section 2 we

\footnotetext{
* Some of the data presented herein were obtained at the W. M. Keck Observatory, which is operated as a scientific partnership among the California Institute of Technology, the University of California, and the National Aeronautics and Space Administration. The Observatory was made possible by the generous financial support of the W. M. Keck Foundation.

6 Present address: Institute of Astronomy, University of Cambridge, Madingley Road, Cambridge CB3 0HA, UK.

7 Present address: Department of Astrophysics, California Institute of Technology, MC 249-17, Pasadena, CA 91125, USA.

8 Present address: School of Physics, Georgia Institute of Technology, 837 State St., Atlanta, GA 30332, USA.
}

describe the observations and data reduction, and in Section 3 we present the model that was used to fit the data and our results for the system parameters. These results are discussed in Section 4.

\section{OBSERVATIONS AND DATA REDUCTION}

\subsection{Photometric Measurements}

Photometric observations of transits were conducted on UT 2008 May 15, June 10, June 21, and UT 2009 May 12 and May 25, using Keplercam on the $1.2 \mathrm{~m}$ telescope at the Fred L. Whipple Observatory (FLWO) on Mount Hopkins, Arizona (Holman et al. 2006). On each night our observations spanned the entire transit, except for the first and second nights when we missed the transit ingress. For the observations in 2008 we used a Sloan $i^{\prime}$ filter, and for those in 2009 we used a Sloan $g^{\prime}$ filter. We processed the Keplercam images with standard IRAF procedures for bias subtraction and flat-field division. Aperture photometry was then performed on WASP-3 and 6-20 nearby comparison stars, and the WASP-3 signal was divided by a normalized sum of the comparison-star signals.

We also observed the complete transit of UT 2008 July 13 with the University of Hawaii 2.2 m (UH 2.2 m) telescope on Mauna Kea, Hawaii. We used the Orthogonal Parallel Transfer Imaging Camera (OPTIC), which is equipped with two Lincoln Labs CCID128 orthogonal transfer array (OTA) detectors (Tonry et al. 1997). Each OTA detector has $2048 \times 4096$ pixels and a scale of 0 .'135 pixel $^{-1}$. We took advantage of the charge-shifting capability of the OTAs to create square point-spread functions (PSF) with side lengths of 39 pixels. This allowed us to collect more light before reaching the saturation limit than is possible in normal imaging mode (Howell et al. 2003; Tonry et al. 2005). We observed through a Sloan $z^{\prime}$ filter, and bias subtraction, flatfielding, and aperture photometry were performed with custom IDL procedures described by Johnson et al. (2009a). 


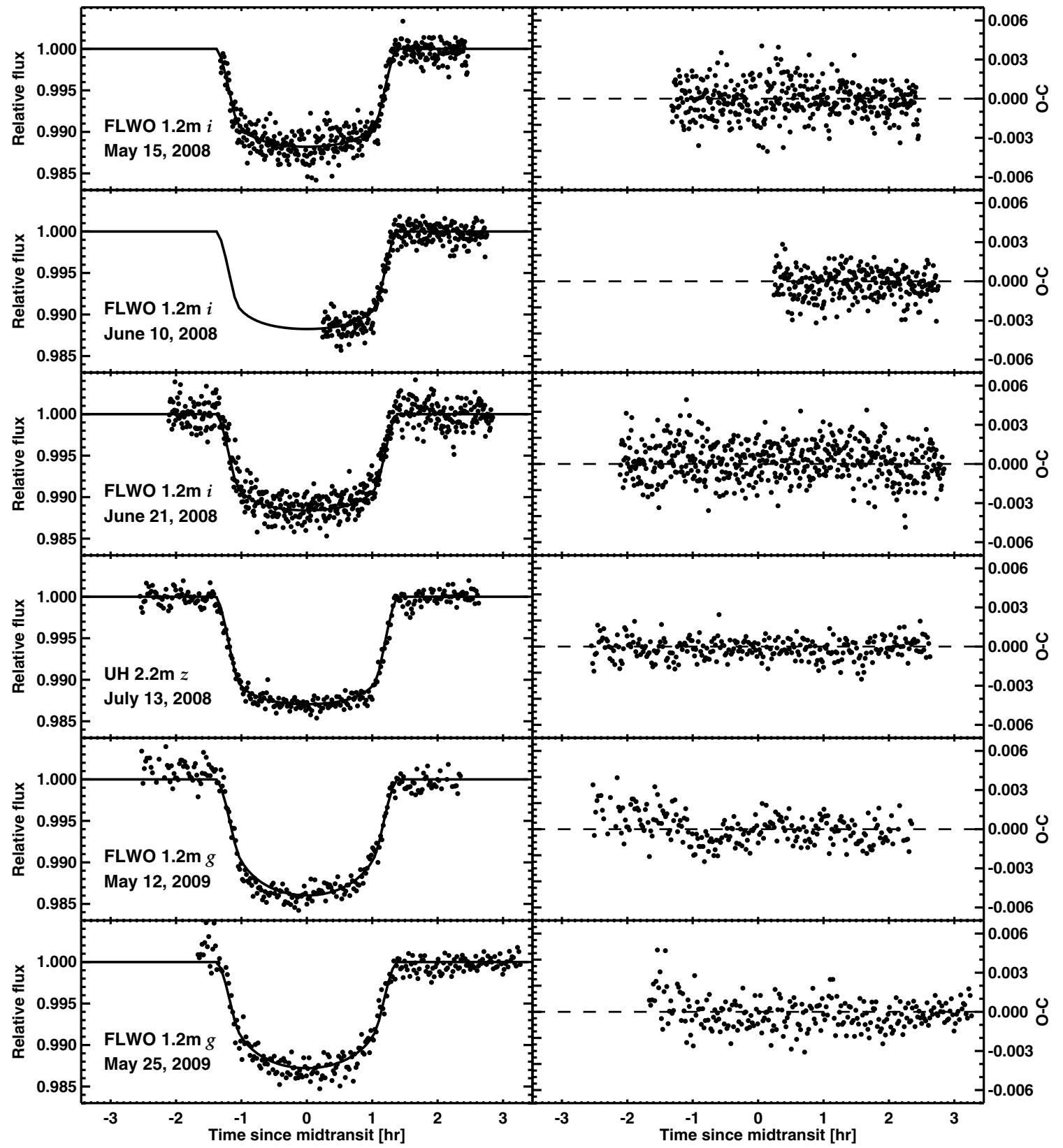

Figure 1. Photometry of transits of WASP-3, based on observations with the FLWO $1.2 \mathrm{~m}$ and UH $2.2 \mathrm{~m}$ telescopes. Left: the data and best-fitting model. Right: residuals between the data and best-fitting model.

Table 1

Relative Photometry of WASP-3

\begin{tabular}{lccc}
\hline \hline Heliocentric Julian Date & Relative Flux & Uncertainty & Observatory \\
\hline 2454601.810140 & 0.99857 & 0.00134 & 1 \\
2454601.810534 & 0.99712 & 0.00134 & 1 \\
2454601.810927 & 0.99775 & 0.00134 & 1 \\
2454601.811321 & 0.99772 & 0.00134 & 1 \\
2454601.811703 & 0.99943 & 0.00134 & 1
\end{tabular}

Note. (1) Fred L. Whipple Observatory $1.2 \mathrm{~m}$ telescope, (2) University of Hawaii $2.2 \mathrm{~m}$ telescope

(This table is available in its entirety in a machine-readable form in the online journal. A portion is shown here for guidance regarding its form and content.)

Final light curves are shown in Figure 1, after correcting for differential extinction as described in Section 3.1. The photometric data are given in Table 1.

\subsection{Radial Velocity Measurements}

We measured the apparent radial velocity (RV) of WASP-3 during the transits of UT 2008 June 19 and 21 with the High Resolution Echelle Spectrometer (HIRES; Vogt et al. 1994) on the Keck I $10 \mathrm{~m}$ telescope at the W. M. Keck Observatory on Mauna Kea. The second of these transits was observed simultaneously with the FLWO $1.2 \mathrm{~m}$ telescope as described in the previous section. We also observed the system with Keck/HIRES on several other nights in 2008 and 2009, all of which were outside of transits except for a single measurement obtained during the transit of UT 2009 June 3.

Our data collection followed the procedure of Johnson et al. (2008) in their measurements of HAT-P-1, and the spectrograph was in the same configuration as used for the California Planet Search (Howard et al. 2009; Marcy et al. 2005). Calibration of the instrumental response and wavelength scale was achieved 
Table 2

Keck/HIRES Doppler Shift Measurements of WASP-3

\begin{tabular}{lrc}
\hline \hline Heliocentric Julian Date & $\begin{array}{c}\text { Radial Velocity } \\
\left(\mathrm{m} \mathrm{s}^{-1}\right)\end{array}$ & $\begin{array}{c}\text { Measurement Uncertainty } \\
\left(\mathrm{m} \mathrm{s}^{-1}\right)\end{array}$ \\
\hline 2454636.833671 & 162.68512 & 6.42080 \\
2454636.878764 & 101.27652 & 6.92383 \\
2454636.899783 & 83.69744 & 7.16853 \\
2454636.908996 & 156.81285 & 7.13308 \\
2454636.918452 & 177.62252 & 6.10846 \\
2454636.930559 & 138.14356 & 7.13066 \\
2454636.940096 & 117.51487 & 6.84713 \\
2454636.951218 & 51.83493 & 6.34730 \\
2454636.959239 & 3.28570 & 6.49503 \\
2454636.969887 & -39.46792 & 7.40002 \\
2454636.980942 & -80.49891 & 6.88382 \\
2454636.991034 & -63.31213 & 7.93881 \\
2454637.001983 & 56.56807 & 8.33331 \\
2454637.014090 & -6.59426 & 6.29524 \\
2454637.060144 & -92.67312 & 7.94984 \\
2454637.090295 & -100.37790 & 6.66956 \\
2454638.816277 & -78.81247 & 7.72656 \\
2454638.825606 & -126.87940 & 7.02801 \\
2454638.836659 & -125.27818 & 7.66176 \\
2454638.845548 & -130.38241 & 6.97449 \\
2454638.854761 & -55.94224 & 7.53164 \\
2454638.865225 & -51.31588 & 7.19155 \\
2454638.877991 & -60.45514 & 7.23436 \\
2454638.890896 & -43.96450 & 6.91079 \\
2454639.006175 & -155.20399 & 6.97899 \\
2454674.740671 & -69.57869 & 7.92163 \\
2454674.744085 & -41.62488 & 9.83204 \\
2454674.868667 & 66.23444 & 8.56491 \\
2454674.994730 & 182.86504 & 9.53239 \\
2454983.909935 & 259.36496 & 9.73989 \\
2454984.890077 & -124.05018 & 8.87455 \\
2454986.041289 & -141.69685 & 8.96584 \\
2454986.920247 & 34.36423 & 8.97649 \\
\hline & & \\
& & \\
& &
\end{tabular}

using an $\mathrm{I}_{2}$ gas absorption cell. The slit width was set by the 0 '.86 B5 decker, and the exposure time ranged from 3 to 5 minutes, giving a resolution of about 60,000 at $5500 \AA$ and a signal-tonoise ratio $(\mathrm{S} / \mathrm{N})$ of approximately $120 \mathrm{pixel}^{-1}$. Doppler shifts were derived from the data using the algorithm of Butler et al. (1996) with subsequent improvements. For a given spectrum, measurement errors were derived from the weighted standard deviation of the mean among the solutions for individual $2 \AA$ spectral segments. The measurement error ranged from 6 to $10 \mathrm{~m} \mathrm{~s}^{-1}$.

The RV data are given in Table 2 and plotted in Figure 2. The figures show the expected sinusoidal variation outside of transits, due to the line-of-sight (LOS) component of the Keplerian orbital velocity of the star, as well as the shortertimescale anomalous RV due to the RM effect that occurs during the transit phase.

In addition to these two sources of RV variability, an unexpected redshift was observed near the egress of the UT 2008 June 19 transit. The RV measured at HJD 2,454,637.001983 is redshifted by $140 \mathrm{~m} \mathrm{~s}^{-1}$ with respect to our best-fitting model (described in Section 3.2). This is much larger than both the estimated measurement error of $8.3 \mathrm{~m} \mathrm{~s}^{-1}$ and the scatter of $\approx 15 \mathrm{~m} \mathrm{~s}^{-1}$ between the other RV data and the best-fitting model. The previous RV data point, at HJD 2,454,636.991034, is not as clearly discrepant but it is the second-largest outlier, with a redshift of $49 \mathrm{~m} \mathrm{~s}^{-1}$ with respect to the best-fitting model. In what follows we will refer to these two consecutive RV data points

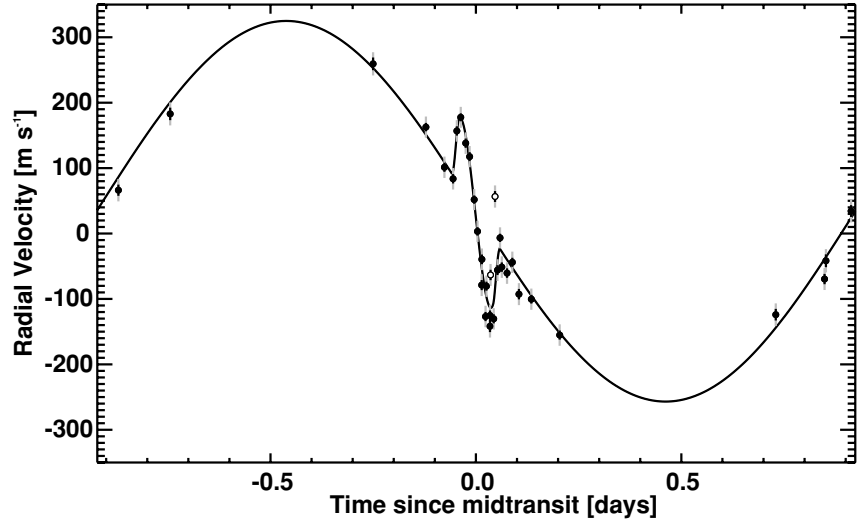

Figure 2. Apparent radial velocity of WASP-3, based on observations with Keck/HIRES. The enlarged (gray) error bars include the "stellar jitter" term of $14.8 \mathrm{~m} \mathrm{~s}^{-1}$ that was added in quadrature with the measurement errors. The small (black) error bars represent the measurement error estimated internally from each particular spectrum. The open symbols represent the "RV spike."

as the "RV spike." They are identified with open symbols in Figure 2. Casual inspection of the spectra constituting the spike revealed nothing unusual. In particular, the visual appearance of the two-dimensional images, the estimated measurement errors, the $\mathrm{S} / \mathrm{N}$ of the spectra, and the goodness-of-fit statistic (and other metrics) returned by the Doppler code were all within normal ranges. Visual examination of the cross-correlation functions (CCFs) did not reveal anything unusual about the spike spectra. The time series of bisector spans was also uninformative, as it shows the same pattern that was already observed in the Doppler shifts (the Rossiter effect and an additional anomaly during the spike). Nevertheless, we believe the spike is an artifact of contamination of the spectra by moonlight, rather than an astrophysical phenomenon, based on the following logic.

1. During our observations, the moon was full and $63^{\circ}$ away from WASP-3. Thin cirrus clouds were noted at approximately the time of the RV spike, and the mean count rates during the "spike" observations declined by approximately a factor of 2. (The exposure times for these spectra were increased to compensate for the reduced count rates.) Thus, the spike spectra were obtained during an interval of reduced transparency and enhanced sky brightness, leading to a possible order-of-magnitude increase in the fractional contamination by moonlight.

2. The moonlight absorption lines would have appeared at a velocity of approximately $+10 \mathrm{~km} \mathrm{~s}^{-1}$ relative to the WASP-3 absorption lines (the difference between the barycentric correction of $4 \mathrm{~km} \mathrm{~s}^{-1}$ and the WASP-3 systemic velocity of $-5.5 \mathrm{~km} \mathrm{~s}^{-1}$ ). This is within the WASP-3 line profiles, which are rotationally broadened to $14 \mathrm{~km} \mathrm{~s}^{-1}$. Given the low contrast $(\approx 10 \%)$ of the WASP-3 lines relative to the continuum, a moonlight contamination of only $\sim 1 \%$ of the total light would be sufficient to produce a $\sim 100 \mathrm{~m} \mathrm{~s}^{-1}$ shift in the center of gravity of the WASP-3 lines. This level of contamination would be visually undetectable in the two-dimensional images.

3 . To assess whether $1 \%$ moonlight contamination is reasonable we used the moonlight model of Krisciunas \& Schaefer (1991), which takes into account the moon phase, star and moon coordinates, and observing bandpass. According to this model, in the absence of clouds the moonlight flux would have been $\approx 0.1 \%$ of the flux of WASP-3. Since the spike spectra were obtained when looking through cirrus, 
it is plausible that the moonlight fraction increased by an order of magnitude to $1 \%$.

Thus, it seems possible and even likely that a small amount of scattered moonlight was responsible for the RV spike. We cannot be certain of this conclusion since we could not devise any conclusive statistical test for moonlight at such low levels, given the simultaneous presence of the RM effect and the time variability of the instrumental broadening profile and the CCFs. For our analysis we omitted the RV spike data from consideration, although we discuss another possible interpretation in Section 4. The investigation of the RV spike was the purpose of the coordinated spectroscopic and photometric observations of the transit of UT 2008 June 21. The RV spike did not recur, and no outliers of similar magnitude were observed.

\section{ANALYSIS AND RESULTS}

\subsection{Photometric Model}

The first step in our analysis was to fit a parametric model to the photometric data. We simplified the analysis by assuming that the planet's orbital eccentricity is zero, which is consistent with previous results (Pollacco et al. 2008; Madhusudhan \& Winn 2009) as well as our own RV observations and the expectation that such a close-in orbit has been tidally circularized. The orbit was parameterized by the period $(P)$, the midtransit time $\left(T_{c}\right)$, the impact parameter $(b)$ in units of the stellar radius, the planet-to-star radius ratio $\left(r \equiv R_{p} / R_{\star}\right)$, and the approximate transit duration $(T)$, defined in Carter et al. (2008) as

$$
T=\frac{P}{\pi b} \sqrt{1-b^{2}} \cos i,
$$

where $i$ is the inclination angle.

The loss of light during the transit was calculated using the analytic formulae of Mandel \& Agol (2002) for a quadratic limb-darkening (LD) law with coefficients $u_{1}$ and $u_{2}$. To speed the convergence of our fitting algorithms, we used the nearly uncorrelated linear combinations of the LD coefficients,

$$
\begin{aligned}
& u_{1}^{\prime}=u_{1} \cos \phi+u_{2} \sin \phi, \\
& u_{2}^{\prime}=u_{2} \cos \phi-u_{1} \sin \phi,
\end{aligned}
$$

with $\phi=39^{\circ}$ (Pál 2008). As a compromise between allowing complete freedom in the LD law and placing complete trust in a theoretical LD law, we allowed $u_{1}^{\prime}$ to be a free parameter and fixed $u_{2}^{\prime}$ at the value predicted from the PHOENIX atmosphere models of Claret (2004). Additionally, as in Carter et al. (2009), we required $u_{1}>0$ and $0<u_{1}+u_{2}<1$ to guarantee that the intensity profile of the star decreased monotonically toward the limb.

In addition, we included two free parameters for each night of observations to specify the out-of-transit magnitude $\left(m_{\mathrm{oot}}\right)$ and a coefficient $(k)$ for differential airmass extinction,

$$
m_{\mathrm{obs}}=m_{\mathrm{oot}}+\Delta m+k z,
$$

where $m_{\mathrm{obs}}$ is the observed magnitude, $\Delta m$ is the calculated magnitude change according to the Mandel \& Agol (2002) formulae, and $z$ is the airmass. As with the LD coefficients, it proved advantageous to use fitting parameters that were appropriate linear combinations of $m_{\text {oot }}$ and $k$.

We fit the data from all six transits, allowing each of the six time series to have independent values of $T_{c}, r, k$, and $m_{\mathrm{oot}}$.
We required consistency in $b$ and $T$, and in the $\mathrm{LD}$ coefficients for all those data sets observed through the same bandpass. However, we did not require consistency in $r$, in order to check for variations in the transit depth that could be caused by a time-variable pattern of starspots or other localized intensity variations across the stellar disk. A time-variable pattern of such irregularities would lead to variations in the transit depth from event to event. For example, when there are dark spots located on the visible hemisphere of the star, the fractional loss of light during transit is greater than when the star is spotless. These transit-to-transit variations would be manifested in our model as an apparent change of the $r$ parameter, even though the actual radii of the bodies are not changing. The idea was to seek any evidence for stellar activity, which would also provide an alternate explanation for the RV spike.

To determine the best values of the parameters and their uncertainties, we used a Markov Chain Monte Carlo (MCMC) algorithm, implemented as described by Holman et al. (2006) and Winn et al. (2007). Specifically, we used a Gibbs sampler and tailored our jump sizes so that they yielded acceptance rates of approximately $40 \%$ for each parameter. The number of links in each of our chains was approximately $10^{5}$ per parameter, so that the distributions for each parameter were well converged. Individual jumps were executed with probability $\exp \left(-\Delta \chi_{f}^{2} / 2\right)$, where

$$
\chi_{f}^{2}=\sum_{i=1}^{N_{f}}\left(\frac{f_{i}(\mathrm{obs})-f_{i}(\text { calc })}{\sigma_{i}}\right)^{2} .
$$

In this expression, $N_{f}$ is the number of data points, $f_{i}$ (obs) is the observed flux, $f_{i}$ (calc) is the flux that is calculated for a given set of model parameters, and $\sigma_{i}$ is a constant for each light curve, determined as follows. First, a value $\sigma_{0}$ was determined such that when using $\sigma_{i}=\sigma_{0}, \chi_{f}^{2}=N_{\text {dof }}$ for the best-fitting model. (The answer was always close to the standard deviation of the out-oftransit data.) Next, we multiplied $\sigma_{0}$ by a factor $\beta$ to account for time-correlated errors, using the time-averaging method of Pont et al. (2006) as implemented by Winn et al. (2009b). Averaging times of 10-30 minutes were used to compute $\beta$, giving results (in chronological order) of $0.92,1.21,1.47,1.30,2.45$, and 1.67. When $\beta$ was found to be smaller than unity, as in the first case, we used $\beta=1$, reasoning that time correlations can only increase errors. Finding $\beta<1$ represents either a statistical fluke or a signal in which the red noise has been underestimated by analyzing the model residuals rather than the out-of-transit data (see Carter \& Winn 2009 for a discussion).

The MCMC analysis was conducted in several stages, taking advantage of the fact that the subset of parameters $\left\{T_{c}, k, m_{\text {oot }}\right\}$ is nearly uncorrelated with the other parameters in the model. First, we determined the midtransit time $\left(T_{c}\right)$ of each event by allowing $T_{c}, k$, and $m_{\text {oot }}$ to be free parameters and fixing all other parameters (which are uncorrelated with $T_{c}$ ) at the values that minimize $\chi_{f}^{2}$. The results for the midtransit times are given in Table 3, along with other previously reported midtransit times.

Our collection of midtransit times was then used to determine the transit ephemeris. The results are given in Table 4 . The linear fit that was used to calculate the ephemeris yielded $\chi^{2}=21$ with 7 degrees of freedom, indicating an unacceptable fit. The transit timing residuals from this fit are shown in Figure 3. Given the large value of $\chi^{2}$, there are either genuine period variations, or the transit time uncertainties have been underestimated in some cases. To be conservative for the purpose of planning future observations, we have increased the errors of our ephemeris 


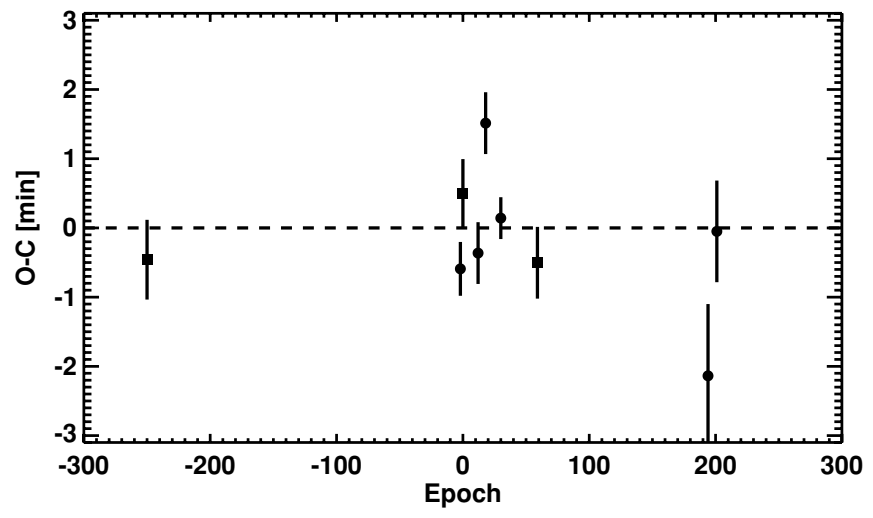

Figure 3. Transit timing residuals. A linear function of epoch was fit to the transit times given in Table 3, and the calculated times were subtracted from the observed times. Circles denote midtransit times from this work; squares denote midtransit times from earlier studies (Pollacco et al. 2008; Gibson et al. 2008).

Table 3

WASP-3 Midtransit Times

\begin{tabular}{lcc}
\hline \hline Epoch & Midtransit Time [HJD] & Reference \\
\hline-250 & $2,454,143.8503 \pm 0.0004$ & 1 \\
-2 & $2,454,601.86514 \pm 0.00027$ & 2 \\
0 & $2,454,605.55956 \pm 0.00035$ & 3 \\
12 & $2,454,627.72098 \pm 0.00031$ & 2 \\
18 & $2,454,638.80329 \pm 0.00031$ & 2 \\
30 & $2,454,660.96435 \pm 0.00021$ & 2 \\
59 & $2,454,714.52210 \pm 0.00036$ & 3 \\
194 & $2,454,963.84361 \pm 0.00072$ & 2 \\
201 & $2,454,976.77290 \pm 0.00051$ & 2 \\
\hline
\end{tabular}

References. (1) Pollacco et al. 2008; (2) this work; (3) Gibson et al. 2008.

values by a factor of $\sqrt{21 / 7}$ above the formal errors of the linear fit.

We determined other system parameters by computing another chain, using our newly determined ephemeris, holding $T_{c}$, $k$, and $m_{\text {oot }}$ fixed at the best-fitting values for each light curve, and allowing all other parameters to vary. The key results are given in Table 4.

Most notable, as mentioned earlier, is the considerable variation in the planet-to-star radius ratio $(r)$, at the $2 \sigma-3 \sigma$ level between the different transits. The mean value of $r$ across all six transits is 0.1059 , from which the individual results differ by as much as $5 \sigma$. The variation in $r$ can also be examined in terms of transit depth $\left(\delta \equiv r^{2}\right)$. The mean of the six $\delta$ values is 0.01122 , and the standard deviation is 0.00080 , which is larger than the statistical uncertainty in any individual depth measurement. As an estimate of the transit-to-transit variation in the transit depth, we sought the value of $\sigma_{\delta}$ such that

$$
\sum_{i=1}^{6} \frac{\left(\delta_{i}-0.01122\right)^{2}}{\sigma_{i}^{2}+\sigma_{\delta}^{2}}=5,
$$

with the result $\sigma_{\delta}=0.00075$. Thus, the data are consistent with fractional variations in the transit depth of order $\sigma_{\delta} / \delta \approx 7 \%$.

Unfortunately, we cannot be confident in the reality of transit depth variations because of the possibility of systematic errors. The light curves shown in Figure 1 clearly exhibit red noise, especially the $g^{\prime}$-band light curves. Our fitting procedure attempts to take the red noise into account but it is inevitably imperfect. It is also suggestive that the $i^{\prime}$-band light curves gave consistent results for $\delta$, while the $z^{\prime}$ and $g^{\prime}$ light curves gave
Table 4

WASP-3 Photometric Model Parameters

\begin{tabular}{|c|c|}
\hline Parameter & Photometric Value \\
\hline Orbital period [d] & $1.846834 \pm 0.000002$ \\
\hline Midtransit time [HJD] & $2454605.55922 \pm 0.00019$ \\
\hline Impact parameter, $b$ & $0.531_{-0.043}^{+0.036}$ \\
\hline Approximate transit duration, $T$ [hr] & $2.412_{-0.015}^{+0.017}$ \\
\hline Transit duration [hr] & $2.813 \pm 0.012$ \\
\hline$R_{p} / R_{\star}\left(\mathrm{UT} 2008\right.$ May $\left.15, i^{\prime}\right)$ & $0.1038_{-0.0011}^{+0.0007}$ \\
\hline$R_{p} / R_{\star}\left(\right.$ UT 2008 June $\left.9, i^{\prime}\right)$ & $0.1041_{-0.0012}^{+0.0008}$ \\
\hline$R_{p} / R_{\star}\left(\mathrm{UT} 2008\right.$ June $\left.21, i^{\prime}\right)$ & $0.1011_{-0.0011}^{+0.0009}$ \\
\hline$R_{p} / R_{\star}\left(\mathrm{UT} 2008\right.$ July $\left.13, z^{\prime}\right)$ & $0.1099_{-0.0010}^{+0.0006}$ \\
\hline$R_{p} / R_{\star}\left(\mathrm{UT} 2009\right.$ May $\left.12, g^{\prime}\right)$ & $0.1107_{-0.0020}^{+0.0014}$ \\
\hline$R_{p} / R_{\star}\left(\mathrm{UT} 2009\right.$ May $\left.25, g^{\prime}\right)$ & $0.1058_{-0.0016}^{+0.0014}$ \\
\hline Limb-darkening coefficient $u_{1}\left(g^{\prime}\right)$ & $0.596_{-0.033}^{+0.031}$ \\
\hline Limb-darkening coefficient $u_{2}\left(g^{\prime}\right)$ & $0.215_{-0.027}^{+0.025}$ \\
\hline Limb-darkening coefficient $u_{1}\left(i^{\prime}\right)$ & $0.288_{-0.035}^{+0.023}$ \\
\hline Limb-darkening coefficient $u_{2}\left(i^{\prime}\right)$ & $0.321_{-0.028}^{+0.019}$ \\
\hline Limb-darkening coefficient $u_{1}\left(z^{\prime}\right)$ & $0.193_{-0.042}^{+0.030}$ \\
\hline Limb-darkening coefficient $u_{2}\left(z^{\prime}\right)$ & $0.283_{-0.036}^{+0.022}$ \\
\hline
\end{tabular}

Notes. The values and uncertainties for $P$ and $T_{c}$ were determined by fitting a straight line to the data given in Table 3 and multiplying the formal errors in the fit by $\sqrt{\chi_{v}^{2}}=\sqrt{3}$. The transit duration is dependent on the radius ratio $\left(R_{p} / R_{\star}\right)$, and hence the value quoted is the mean and standard deviation of the six transit durations determined (using each of the $R_{p} / R_{\star}$ values). For the other parameters, the quoted value represents the mode of the MCMC distribution, and the quoted uncertainties are " $1 \sigma$ " errors, spanning the range between the $15.85 \%$ and $84.15 \%$ levels of the MCMC cumulative distribution. One final note is that the approximate transit duration $(T)$ refers to the quantity defined in Equation (1), making it different from the transit duration.

larger values for $\delta$; this might be due to systematic errors in the treatment of LD.

Comparison with previous studies reveals that we found a larger transit depth than Pollacco et al. (2008) and, similarly, a larger radius ratio than Gibson et al. (2008). Our results for these parameters disagree with each of the two studies by $3 \sigma$ and $5 \sigma$, respectively. Of our other photometric parameters, we find agreement with the values that were reported by Pollacco et al. (2008), but less so with those reported by Gibson et al. (2008). The latter authors reported a more precise result for $b$ of $0.448 \pm 0.014$ that disagrees with our result of $0.531_{-0.043}^{+0.036}$. They also found a shorter transit duration, $2.753_{-0.013}^{+0.020} \mathrm{hr}$, compared to our result of $2.813 \pm 0.012 \mathrm{hr}$. The reason for these discrepancies is unclear, but we suspect that at least part of the reason is that the Gibson et al. (2008) uncertainties were underestimated because the LD law was held fixed.

\subsection{Radial Velocity Model}

We fitted the RV data with a parametric model that takes into account both the orbital velocity of the star $\left(V_{o}\right)$ and the anomalous velocity due to the RM effect $\left(\Delta V_{r}\right)$. As in our photometric model, we maintained the assumption of a circular orbit. Our orbital model was parameterized by the period $(P)$, the midtransit time $\left(T_{c}\right)$, the velocity semi-amplitude $(K)$, and a constant velocity offset $(\gamma)$. The offset parameter was needed because the precise Doppler velocities were computed with respect to a template spectrum with an arbitrary velocity zero point. Furthermore, as we will describe shortly, we included an additional velocity offset $(\Delta \gamma)$ specific to the observations that were made on UT 2008 June 19. 
The RM effect was parameterized by the projected spin-orbit angle $(\lambda)$ and the LOS stellar rotational velocity $\left(v \sin i_{\star}\right)$. To calculate $\Delta V_{r}$ we used the "RM calibration" procedure of Winn et al. (2005): we simulated spectra exhibiting the RM effect at different phases of the transit and then measured the apparent RV of the simulated spectra. ${ }^{9}$ We found the results to be consistent with the formula

$$
\Delta V_{r}=-(\Delta f) V_{p}\left[1.51-0.44\left(\frac{V_{p}}{10 \mathrm{~km} \mathrm{~s}^{-1}}\right)^{2}\right],
$$

where $\Delta f$ is the fractional loss of light during the transit and $V_{p}$ is the LOS component of the stellar rotation velocity at the location hidden by the planet.

Since $\Delta V_{r}$ depends on $\Delta f$, the RV model is dependent on the photometric parameters. To determine these parameters, one could fit the RV and photometric data simultaneously, but we found it faster and more convenient to fit the RV data alone using prior constraints on the relevant photometric parameters. Specifically we used $b, T$, and $r$ as parameters in the RV model, the first two of which were subjected to Gaussian prior constraints from the photometry. As for $r$, given the discrepancies among the results of fitting individual transits, we adopted a Gaussian prior with a central value of 0.1049 and an uncertainty of 0.0037 , reflecting the median and standard deviation of the six different results.

Thus, our RV fitting statistic was

$$
\begin{aligned}
\chi_{v}^{2}= & \sum_{i=1}^{N_{v}}\left(\frac{v_{i}(\mathrm{obs})-v_{i}(\mathrm{calc})}{\sigma_{i}}\right)^{2}+\left(\frac{b-0.531}{\sigma_{b}}\right)^{2} \\
& +\left(\frac{T-2.412 \mathrm{hr}}{\sigma_{T}}\right)^{2}+\left(\frac{r-0.1049}{0.0037}\right)^{2},
\end{aligned}
$$

where $N_{v}$ is the number of RV data points, $v_{i}$ (obs) is the observed RV, $v_{i}$ (calc) is the RV that is calculated for a given set of model parameters, and $\sigma_{b}$ and $\sigma_{T}$ are taken from Table 4 depending on the sign of the difference in the numerator. The LD coefficients were fixed at the values tabulated by Claret (2004) for the PHOENIX model in the $g^{\prime}$ bandpass (where we find the strongest signal from the $I_{2}$ absorption lines used for RV data calibration). The period and midtransit time were fixed at the values determined in Section 3.1.

The two measurements constituting the RV spike of UT 2008 June 19, described in Section 2.2, were significant outliers that did not conform to our model, regardless of the parameter values that were chosen. Assuming that they were influenced by a physical process that was not described by our model — whether moonlight, as we discussed in Section 2, or an astrophysical phenomenon-we omitted these two data points from the fit. We also omitted a third RV point immediately following the spike, even though this data point was not an outlier, because it was based on observations through cirrus clouds and was thus suspected of being affected by moonlight. (None of the results we describe subsequently are altered materially if this third point is instead included in the fit.) Furthermore, we allowed the data from UT 2008 June 19 to have an extra velocity offset $(\Delta \gamma)$, in addition to the velocity offset $(\gamma)$ applied to all of

\footnotetext{
9 The starting point of the simulations was a spectrum from Allende-Prieto (2002) of Procyon (F5, $T_{\text {eff }}=6500 \mathrm{~K}$ ) that was chosen because it is a slower rotator than WASP-3 but its spectrum is similar in other respects. For details on the construction of the simulated RM spectra, refer to Winn et al. (2005).
}

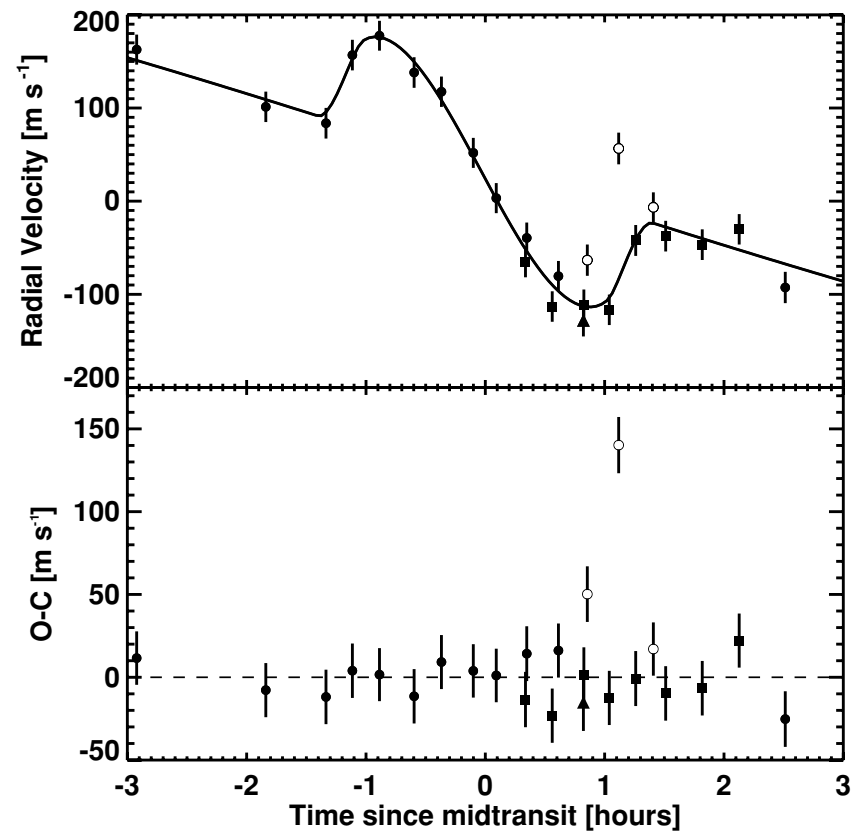

Figure 4. Spectroscopic transit of WASP-3. Top: the RV data from UT 2008 June 19 (circles), 2008 June 21 (squares), and 2009 June 3 (triangle). The open symbols show the data which were omitted from the fit: the two points of the RV spike, and the next data point, which is also anomalously redshifted although by a much smaller degree. The constant velocity offsets given in Table 5 have been applied and the error bars shown here include stellar jitter. Bottom: residuals between the data and the best-fitting model.

the RV data. We incorporated this additional offset to allow for any systematic offset of the data from that night, again due to moonlight or astrophysical phenomenon responsible for the RV spike.

Taking $\sigma_{i}$ to be the measurement uncertainty given in Table 2, the minimum $\chi_{v}^{2}$ was 127 with 28 degrees of freedom, an unacceptable fit. We enlarged the RV errors by adding $14.8 \mathrm{~m} \mathrm{~s}^{-1}$ in quadrature with the measurement errors in order to achieve a reduced $\chi_{v}^{2}$ of unity. The excess scatter is referred to as "stellar jitter" and is attributed to intrinsic motions of the stellar photosphere, unmodeled orbital motions (due to additional planets or companion stars) and unknown systematic errors. This procedure of adding in stellar jitter is common in fitting high-precision RV data, and the value of $14.8 \mathrm{~m} \mathrm{~s}^{-1}$ is in line with previous observations of F stars rotating as rapidly as WASP-3 (Saar et al. 1998).

Figure 4 shows the RV data, with the omitted points highlighted, the error bars enlarged to encompass the stellar jitter, and the velocity offsets applied. Results from the fit are given in Table 5 . We find the parameters describing the RM effect to be $v \sin i_{\star}=14.1_{-1.3}^{+1.5} \mathrm{~km} \mathrm{~s}^{-1}$ and $\lambda=3.3_{-4.4}^{+2.5} \mathrm{deg}$. Our result for $v \sin i_{\star}$ is in agreement with the value of $13.4 \pm 1.5 \mathrm{~km} \mathrm{~s}^{-1}$ that was previously reported by Pollacco et al. (2008), based on an analysis of the spectral line profiles rather than on the RM effect. The consistency between these results lends confidence to both analyses and our "RM calibration" procedure. Our result for $\lambda$ is new information about this planetary system, indicating good alignment between the stellar rotation axis and the projected orbit normal. Our result for the velocity semiamplitude $K$ is $290.5_{-9.2}^{+9.8} \mathrm{~m} \mathrm{~s}^{-1}$, which is about $3 \sigma$ larger than the value of $251.2_{-10.8}^{+7.9} \mathrm{~m} \mathrm{~s}^{-1}$ reported by Pollacco et al. (2008), but which is in agreement with the more recent determination of $274 \pm 11 \mathrm{~m} \mathrm{~s}^{-1}$ by Simpson et al. (2010). 
Table 5

WASP-3 RV Model Parameters

\begin{tabular}{lc}
\hline \hline \multicolumn{1}{c}{ Parameter } & Value \\
\hline$v \sin i_{\star}\left(\mathrm{km} \mathrm{s}^{-1}\right)$ & $14.1_{-1.3}^{+1.5}$ \\
$\lambda(\mathrm{deg})$ & $3.3_{-4.4}^{+2.5}$ \\
$K\left(\mathrm{~m} \mathrm{~s}^{-1}\right)$ & $290.5_{-9.2}^{+9.8}$ \\
$M_{\mathrm{p}}\left(M_{\mathrm{J}}\right)^{\mathrm{a}}$ & $2.04_{-0.07}^{+0.07}$ \\
$\gamma\left(\mathrm{m} \mathrm{s}^{-1}\right)$ & $33.5_{-4.5}^{+6.3}$ \\
$\Delta \gamma\left(\mathrm{m} \mathrm{s}^{-1}\right)$ & $14.1_{-6.9}^{+6.2}$
\end{tabular}

Note.

assuming $M_{\star}=1.24 M_{\odot}$ per Pollacco et al. (2008), and $i=84.1$, as determined from our light-curve analysis.

\section{DISCUSSION}

We have observed the RM effect during transits of WASP-3b and found that the projected spin-orbit angle is $\lambda=3.3_{-4.4}^{+2.5} \mathrm{deg}$, suggesting that the stellar rotation axis and the orbital axis are closely aligned. ${ }^{10}$ This alignment makes WASP-3 similar to the majority of other exoplanets whose alignment has been studied (Fabrycky \& Winn 2009) and unlike the misaligned systems XO-3 (Hébrard et al. 2008; Winn et al. 2009c), HD 80606 (Pont et al. 2009; Winn et al. 2009d), WASP-14 (Johnson et al. 2009b), HAT-P-7 (Narita et al. 2009; Winn et al. 2009a), Corot-1 (Pont et al. 2009), and WASP-17 (Anderson et al. 2010). It would seem that for WASP-3, the inward migration process did not disrupt the initial coplanarity of the system, or tidal effects have coplanarized the system (although the theoretically calculated rate of coplanarization is too slow to be relevant, Barker \& Ogilvie 2009).

We also used new RV measurements and transit light curves to determine the system parameters, and found agreement with previously published values, with the exception of the RV semiamplitude $K$ reported by Pollacco et al. (2008). In addition, we observed an intriguing RV anomaly during one of the transits. Our investigation of the spectra led us to the conclusion that this "RV spike" was caused by a small amount of moonlight that was admitted to the slit along with the light from WASP-3. However, we cannot be completely certain in this interpretation, and in any case it is interesting to ask whether there are any astrophysical phenomena that could produce such an effect.

One speculative idea is that the spike represents the planet's passage in front of a starspot. When a spot is hidden by a planet, the apparent RV of the star will jump to a larger or smaller value, because the RM-like effect of the spot temporarily vanishes. When the spot is no longer hidden, the star's apparent RV returns to its expected transiting value. The resulting "spike" in RV would be analogous to the photometric "bumps" or "rebrightenings" that have been seen in transit light curves and attributed to starspot crossings, as in Rabus et al. (2009) and Dittmann et al. (2009), amongst others.

The time between the RV spike's initial point and its maximum $(\approx 20$ minutes) is consistent with the ingress or egress duration, as would be required of the spot-occultation hypothesis. However, to match the $+140 \mathrm{~m} \mathrm{~s}^{-1}$ amplitude of the RV spike, the starspot would need to present a velocity contrast with

\footnotetext{
10 While the first draft of this manuscript was under review, we learned of a similar study of WASP-3 by Simpson et al. (2010). They also measured the $\mathrm{RM}$ effect, and found $\lambda=15_{-9}^{+10} \mathrm{deg}$, also suggesting a relatively close spin-orbit alignment.
}

the photosphere, in addition to a possible intensity contrast. This can be understood as follows: under the starspot hypothesis the amplitude of the RV spike would have an amplitude

$$
\Delta v \approx v_{\star}\left(\frac{R_{s}}{R_{\star}}\right)^{2}\left(1-\frac{I_{s} v_{s}}{I_{\star} v_{\star}}\right),
$$

assuming there is a single spot with radius $R_{S}$, intensity $I_{S}$, and radial velocity $v_{s}$ that may differ from the surrounding photosphere. In the absence of the spot, the material at the spot's location would have intensity $I_{\star}$ and radial velocity $v_{\star}$. Because the spike occurred near the transit egress, the spot would have been near the receding limb of the star, where $v_{\star}=+v \sin i_{\star} \sqrt{1-b^{2}} \approx+12 \mathrm{~km} \mathrm{~s}^{-1}$. Assuming $R_{s} \approx R_{p}$ and setting $\Delta v$ of Equation (9) equal to $140 \mathrm{~m} \mathrm{~s}^{-1}$, we find

$$
\frac{I_{s} v_{s}}{I_{\star} v_{\star}} \approx-0.06
$$

Since intensity cannot be negative, the above relation implies that the spot's effective RV must be oppositely directed from the local photospheric RV. The planet must cover a blueshifted spot in order to produce a redshift as large as $140 \mathrm{~m} \mathrm{~s}^{-1}$. Specifically, the spot's peculiar $\mathrm{RV}$, defined as $v_{s}-v_{\star}$, is approximately $-12.7 \mathrm{~km} \mathrm{~s}^{-1}\left(I_{\star} / I_{s}\right)$. Unless the spot is very bright $\left(I_{s} \gg I_{\star}\right)$ the peculiar velocity just estimated would exceed the sound speed of the photosphere, which we estimate to be $7 \mathrm{~km} \mathrm{~s}^{-1}$. The hidden-spot hypothesis for the RV spike thus requires not an ordinary spot, but rather an erupting and possibly even supersonic spot. What we are calling a "spot" would actually be more akin to an active site undergoing a stellar flare.

Thus, the RV anomaly could be rationalized as a consequence of vigorous stellar activity, a hypothesis which would also produce transit depth variations (see Section 3.1). A serious objection to this hypothesis, however, is that by the usual measures WASP-3 is a quiescent main-sequence F7-8 dwarf star for which stellar activity is not expected. Pollacco et al. (2008) presented no evidence for photometric variations, chromospheric emission lines, or any other indication of stellar activity. Likewise, there is no detectable $\mathrm{Ca}$ II $\mathrm{H} \& \mathrm{~K}$ emission in any of our own Keck/HIRES spectra, including those obtained during the RV spike. The measured Mt. Wilson $S$ values had an rms of only 0.0013 , with no noticeable offset even between the measurements spanning different seasons. Considering $R_{\mathrm{HK}}^{\prime}$, the ratio of chromospheric emission in $\mathrm{Ca}$ II $\mathrm{H} \& \mathrm{~K}$ cores to the total stellar emission, we find that WASP-3's mean $\log R_{\mathrm{HK}}^{\prime}=-4.9$, giving it an approximate age of 4 Gyr (Noyes et al. 1984). By comparison, a very active star such as HD 189733 has $\log R_{\mathrm{HK}}^{\prime}=-4.5$, while a very quiet star such as HD 9407 has $\log R_{\mathrm{HK}}^{\prime}=-4.98$ (Wright et al. 2004). Finally, one might expect an unusually active star to be an unusually rapid rotator, but the value of $v \sin i_{\star}=14.1 \mathrm{~km} \mathrm{~s}^{-1}$ is typical of an F7V star. The activity hypothesis would therefore imply a high level of activity in a star that one would otherwise expect to be quiescent.

Although the activity hypothesis is not tenable for WASP-3, we predict that similar observations of other systems with more active stars will eventually reveal "RV spikes" when the planet occults active regions. Simultaneous photometry and spectroscopy of these events will allow investigations of the intensity and velocity contrast of the active regions, relative to the surrounding photosphere. Active stars are often excluded from Doppler planet surveys, precisely because the excess RV noise caused by stellar activity is a hindrance to planet detection. However, magnitude-limited photometric transit surveys have 
no such selection, and in this regard it is unsurprising that planets around very active stars are being routinely discovered by these surveys, such as CoRoT-2 (Lanza et al. 2009) and CoRoT-7 (Léger et al. 2009). The Doppler follow-up for these systems will undoubtedly be more difficult than for quiescent stars. But, perhaps these difficulties will be compensated to some degree by the prospect that active stars with eclipsing planets will eventually enable a deeper understanding of starspots, stellar activity, and stellar flares.

We thank N. Madhusudhan, Michael L. Stevens, and Robert Noyes for helpful discussions. We are grateful to the anonymous referee for helping to clarify the interpretation of the RV spike. The authors wish to recognize and acknowledge the very significant cultural role and reverence that the summit of Mauna Kea has always had within the indigenous Hawaiian community. We are most fortunate to have the opportunity to conduct observations from this mountain. A.T. and K.dK. thank the MIT UROP Endowment Fund and Office for financial support. J.N.W. is grateful to the NASA Origins program for support through grants NNX09AD36G and NNX09AB33G, and to the MIT Class of 1942 for a Career Development Professorship. J.A.J. is an NSF Astronomy and Astrophysics Postdoctoral Fellow with support from the NSF grant AST-0702821.

Facilities: FLWO:1.2m, UH:2.2m, Keck:I

\section{REFERENCES}

Allende-Prieto, C. 2002, HST Proposal, 9368

Anderson, D. R., et al. 2010, ApJ, 709, 159

Barker, A. J., \& Ogilvie, G. I. 2009, MNRAS, 395, 2268

Butler, R. P., Marcy, G. W., Williams, E., McCarthy, C., Dosanjh, P., \& Vogt, S. S. 1996, PASP, 108, 500

Carter, J. A., \& Winn, J. N. 2009, ApJ, 704, 51

Carter, J. A., Winn, J. N., Gilliland, R., \& Holman, M. J. 2009, ApJ, 696, 241

Carter, J. A., Yee, J. C., Eastman, J., Gaudi, B. S., \& Winn, J. N. 2008, ApJ, 689,499

Claret, A. 2004, A\&A, 428, 1001

Dittmann, J. A., Close, L. M., Green, E. M., \& Fenwick, M. 2009, ApJ, 701, 756
Fabrycky, D. C., \& Winn, J. N. 2009, ApJ, 696, 1230

Gaudi, B. S., \& Winn, J. N. 2007, ApJ, 655, 550

Gibson, N. P., et al. 2008, A\&A, 492, 603

Giménez, A. 2006, ApJ, 650, 408

Hébrard, G., et al. 2008, A\&A, 488, 763

Holman, M. J., et al. 2006, ApJ, 652, 1715

Howard, A. W., et al. 2009, ApJ, 696, 75

Howell, S. B., Everett, M. E., Tonry, J. L., Pickles, A., \& Dain, C. 2003, PASP, 115,1340

Johnson, J. A., Winn, J. N., Cabrera, N. E., \& Carter, J. A. 2009a, ApJ, 692, L100

Johnson, J. A., et al. 2008, ApJ, 686, 649

Johnson, J. A., et al. 2009b, PASP, 121, 1104

Krisciunas, K., \& Schaefer, B. E. 1991, PASP, 103, 1033

Lanza, A. F., et al. 2009, A\&A, 493, 193

Léger, A., et al. 2009, A\&A, 506, 287

Madhusudhan, N., \& Winn, J. N. 2009, ApJ, 693, 784

Mandel, K., \& Agol, E. 2002, ApJ, 580, L171

Marcy, G. W., Butler, R. P., Vogt, S. S., Fischer, D. A., Henry, G. W., Laughlin, G., Wright, J. T., \& Johnson, J. A. 2005, ApJ, 619, 570

Narita, N., Sato, B., Hirano, T., \& Tamura, M. 2009, PASJ, 61, L35

Noyes, R. W., Hartmann, L. W., Baliunas, S. L., Duncan, D. K., \& Vaughan, A. H. 1984, ApJ, 279, 763

Ohta, Y., Taruya, A., \& Suto, Y. 2005, ApJ, 622, 1118

Pál, A. 2008, MNRAS, 390, 281

Pollacco, D., et al. 2008, MNRAS, 385, 1576

Pont, F., Zucker, S., \& Queloz, D. 2006, MNRAS, 373, 231

Pont, F., et al. 2009, A\&A, 502, 695

Queloz, D., Eggenberger, A., Mayor, M., Perrier, C., Beuzit, J. L., Naef, D., Sivan, J. P., \& Udry, S. 2000, A\&A, 359, L13

Rabus, M., et al. 2009, A\&A, 494, 391

Saar, S. H., Butler, R. P., \& Marcy, G. W. 1998, ApJ, 498, L153

Simpson, E. K., et al. 2010, MNRAS, 548

Tonry, J., Burke, B. E., \& Schechter, P. L. 1997, PASP, 109, 1154

Tonry, J. L., Howell, S. B., Everett, M. E., Rodney, S. A., Willman, M., \& VanOutryve, C. 2005, PASP, 117, 281

Vogt, S. S., et al. 1994, Proc. SPIE, 2198, 362

Winn, J. N., et al. 2005, ApJ, 631, 1215

Winn, J. N., Holman, M. J., \& Roussanova, A. 2007, ApJ, 657, 1098

Winn, J. N., Johnson, J. A., Albrecht, S., Howard, A. W., Marcy, G. W., Crossfield, I. J., \& Holman, M. J. 2009a, ApJ, 703, L99

Winn, J. N., et al. 2009b, ApJ, 693, 794

Winn, J. N., et al. 2009c, ApJ, 700, 302

Winn, J. N., et al. 2009d, ApJ, 703, 2091

Wright, J. T., Marcy, G. W., Butler, R. P., \& Vogt, S. S. 2004, ApJS, 152, 261 